\title{
Primary hepatic sarcomatoid carcinoma: A case report
}

\author{
QIANYING LENG $^{1,2}$, XI XIANG ${ }^{1}$, YUANJIAO TANG ${ }^{1}$, YUJIA YANG ${ }^{1}$ and LI QIU ${ }^{1}$ \\ ${ }^{1}$ Department of Ultrasound, West China Hospital of Sichuan University; ${ }^{2}$ Department of Ultrasound, No.4 \\ West China Teaching Hospital of Sichuan University, Chengdu, Sichuan 610041, P.R. China
}

Received August 25, 2014; Accepted June 1, 2015

DOI: $10.3892 /$ etm.2015.2599

\begin{abstract}
Sarcomatoid carcinoma (SC) is a malignant tumor type of unclear pathogenesis, which rarely occurs in the liver. The present study reports the case of a 60-year-old woman who presented with multiple masses in her liver, with no fever, abdominal pain or jaundice. Serological markers for hepatitis $\mathrm{B}$ and $\mathrm{C}$ virus were negative, as was the test for $\alpha$ fetoprotein. Abdominal enhanced magnetic resonance imaging examination confirmed multiple lesions in the liver, and liquefactive necrosis was observed. The patient underwent a right hepatectomy, in addition to a cholecystectomy. Cytokeratin 8 (CK8), cluster of differentiation 117, pancytokeratin and vimentin were positively detected using immunohistochemistry, and thus the diagnosis of primary hepatic SC (PHSC) was established. During the follow-up period, characteristic imaging manifestations of tumor recurrence were detected, including peripheral enhancement, central necrosis, variable enhancement, venous invasion and intrahepatic metastasis. The immunohistochemical detection of CK, epithelial membrane antigen and vimentin, with CK8 as a critical indicator, may be useful for the diagnosis of PHSC. The preferred treatment for PHSC is surgical resection and the prognosis is poor.
\end{abstract}

\section{Introduction}

Sarcomatoid carcinoma (SC), also known as spindle cell carcinoma, pseudosarcoma and carcinosarcoma, is a malignant tumor type of unclear pathogenesis. Previous studies have reported cases of SC in the skin, breast, urinary tract, lung, head and neck mucosal region and small intestine (1-6). $\mathrm{SC}$ rarely occurs in the liver, and has been detected in only $1.8 \%$ of all surgically resected hepatocellular carcinomas (HCCs) and in 3.9-9.4\% of autopsied cases $(7,8)$. To the best of our knowledge, the majority of hepatic SC (HSC) cases

Correspondence to: Mrs Li Qiu, Department of Ultrasound, West China Hospital of Sichuan University, 37 Guo Xue Xiang, Chengdu, Sichuan 610041, P.R. China

E-mail: wsqiuli@126.com

Key words: sarcomatoid carcinoma, primary hepatic sarcomatoid carcinoma reported in the English literature occurred with a simultaneous HCC or cholangiocellular carcinoma (CCC). With regard to the histogenesis of $\mathrm{HSC}$, it has been suggested that sarcomatoid elements in liver carcinomas are derived from a dedifferentiation of HCC (7). HCC and CCC exhibit a number of histological variations, and anaplastic sarcomatoid changes are known to arise with a reported frequency of 2.2-2.9\% (9). Local recurrence, venous invasion and intrahepatic, distant and lymph node metastasis occur frequently. The case reported in the present study possessed two noteworthy characteristics: First, it was a rare case of pure primary HSC (PHSC). Secondly, the recurrence of the tumor was recorded by imaging. The present study reports the primary clinical, pathological and immunohistochemical observations of the case, as well as the treatment and detection of tumor recurrence.

\section{Case report}

A 60-year-old woman was admitted to the West China Hospital of Sichuan University (Chengdu, China) with multiple masses in her liver, but with no complaints of fever, abdominal pain, jaundice or other symptoms. Serological markers for hepatitis $\mathrm{B}$ and $\mathrm{C}$ virus were negative, as was the test for $\alpha$ fetoprotein (AFP; $1.99<8 \mathrm{ng} / \mathrm{ml}$ ). An abdominal enhanced magnetic resonance imaging (MRI) examination confirmed multiple lesions in the liver; these were predominantly located in the right lobe, with evident liquefactive necrosis (Fig. 1). Further physical examination and other tests confirmed that no lesions existed elsewhere in the body. The patient subsequently underwent a right hepatectomy and cholecystectomy. Intraoperatively, three hard lesions were detected; these lesions were $\sim 3.0 \mathrm{~cm}$ in diameter and were encapsulated in segments 7 and 8 of the liver. Overall, the resected specimens measured $14 \times 10 \times 6.5 \mathrm{~cm}$, which included multiple gray nodules with a diameter of $0.5-3 \mathrm{~cm}$. No obvious cirrhosis was observed surrounding the lesions. Furthermore, there were no indications of ordinary HCC and CCC in the resected specimens, and the surgical margins were free of tumor. Histologically, the majority of the regions consisted of spindle-cell components, while the epithelial-cell components exhibited a focal distribution. Immunohistochemically, positive results were obtained for cytokeratin 8 (CK8), pancytokeratin, cluster of differentiation (CD) 117 and vimentin; however, the sample was negative for Discovered on GIST-1, CD34, synaptophysin and chromogranin A. The positive rate 


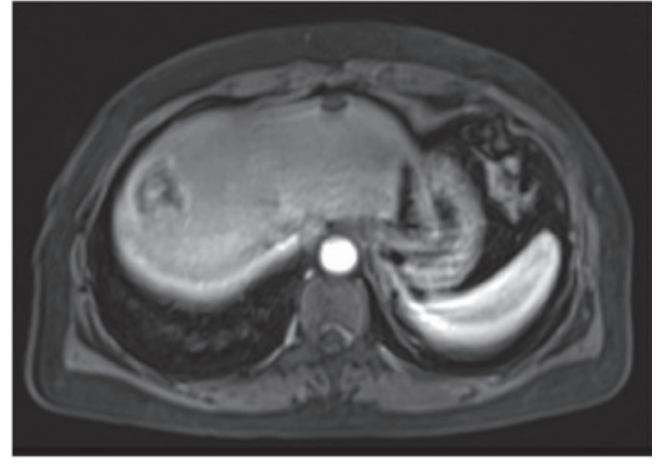

Figure 1. Enhanced magnetic resonance imaging revealed lesions with liquefaction and necrosis in the center, with peripheral enhancement in the liver.
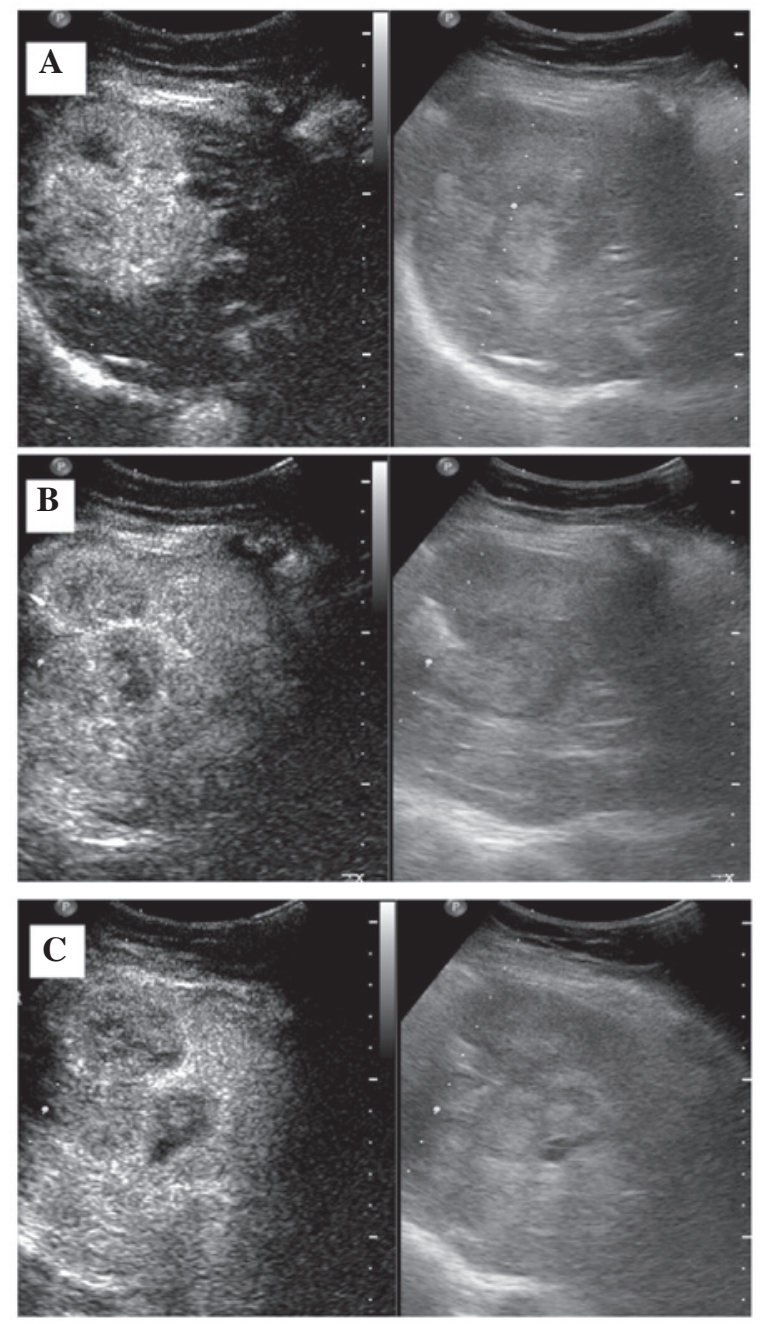

Figure 2. Contrast-enhanced ultrasound images in the (A) arterial phase, showing rapid, marked enhancement, and (B) the portal and (C) the delayed phases, showing limited enhancement.

of Ki-67 detection was $\sim 50 \%$. No hepatocytes were observed in the resected samples. The results of a fluorescence in situ hybridization test indicated no SS18 gene translocation. The result of the KIT/PDGFRA mutation test indicated no KIT exon 9 and 13 mutations or PDGFRA exon 14 and 18 mutations. Finally, a diagnosis of poorly differentiated PHSC was established. Postoperatively, the patient received thymosin
A

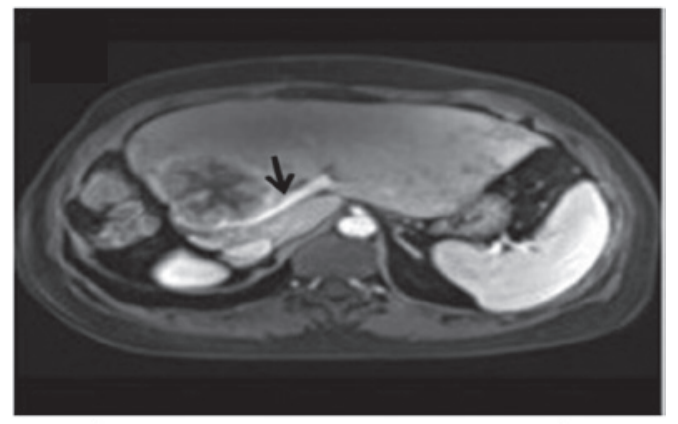

B

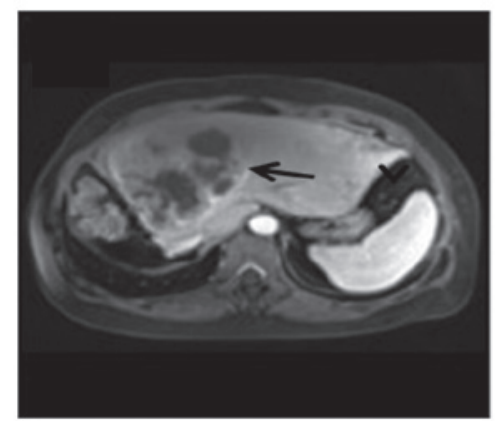

C

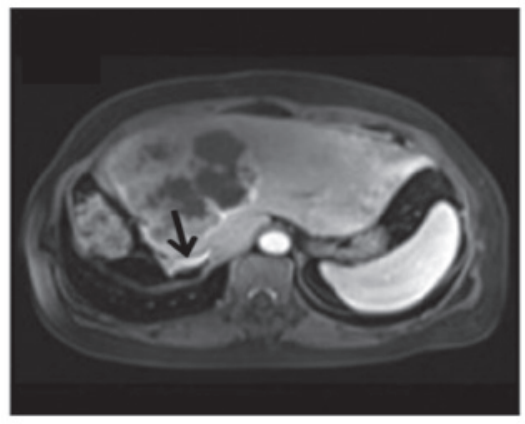

Figure 3. Vascular invasions visualized using enhanced magnetic resonance imaging. (A) The left portal vein exhibited no clear boundaries with the tumor; (B) the left hepatic vein was not clear and (C) the inferior vena cava became narrow due to mass compression.

(80 mg qd) treatment, but no radiotherapy or chemotherapy was administered.

After a 3-month follow-up period, conventional ultrasound examination indicated a weak-echo mass of $2 \mathrm{~cm}$ in the residual liver. Two months later, the mass had developed into a $6.1 \times 6.4-\mathrm{cm}$ clutter-echo mass in the residual liver, and exhibited an irregular internal echo-free zone under conventional ultrasound. Contrast-enhanced ultrasound (CEUS) was immediately conducted, which revealed that the mass was rapidly and markedly enhanced in the arterial phase, while weakly enhanced in the portal and delayed phases (Fig. 2). Thus, tumor recurrence was diagnosed and the patient received chemotherapy as opposed to surgical treatment. After $\sim 6$ months, the patient was referred for a further review. An MRI examination revealed that the residual liver was enlarged, and multiple nodules and masses with unequal size were evident, with a reduced $\mathrm{T} 1$ signal and an elevated T2 signal. The largest mass, which was lobulated and had a maximum cross-section of $\sim 10.3 \times 10.7 \mathrm{~cm}$, was located in the left inner lobe. Enhanced MRI scanning indicated that the lesions were ring-enhanced, with no enhancement of central necrosis. The largest lesion had no clear boundaries with the inferior vena cava near the second hepatic hilum or the left 
branch of the portal vein trunk. The left hepatic vein mixed with the left external lobe lesions (Fig. 3). Thus, the recurrence and widespread intrahepatic metastasis of the PHSC was confirmed. Furthermore, the tumors had invaded the inferior vena cava, near the second hepatic hilum, left portal vein trunk and left hepatic vein. The patient and her family elected to forgo further treatment and the patient succumbed to the PHSC 2 months later due to her poor general condition. Written, informed consent was obtained from the patient's family.

\section{Discussion}

PHSC is a rare malignant tumor type of unclear pathogenesis. The present study reports a rare case of pure PHSC with a rapid clinical course; HSC has been reported in $1.8 \%$ of all surgically resected HCCs and in $3.9-9.4 \%$ of the autopsied cases $(7,8)$. Using immunohistochemistry, Kakizoe et al (7) concluded that the appearance of sarcomatoid components represented a sarcomatoid change in the $\mathrm{HCC}$, rather than a complication of HCC and sarcoma. Thus, a diagnosis of sarcomatoid change in $\mathrm{HCC}$ is feasible based on previous literature, particularly for patients with HCC following artery embolization, anticancer treatment, radio frequency ablation or liver transplantation (10-12). These patients usually possess a history of hepatitis B or C, or a foundation disease of liver cirrhosis due to various causes, such as alcohol. The present case, by contrast, did not present with these features (13). Comparable with ordinary HCC, the clinical symptoms associated with PHSC are typically abdominal pain, weight loss, anorexia and fatigue; however, fever and abdominal pain are considered to be more specific manifestations of SC (14). The AFP expression levels in HSC patients may be normal or slightly elevated; this differs from HCC, in which AFP is considered to be a characteristic diagnostic indicator.

Imaging examinations serve a crucial function in the diagnosis of PHSC. PHSC typically presents as a mass with peripheral enhancement, central necrosis, variable enhancement of the solid portion with or without tumor capsule, and intrahepatic metastasis in enhanced computed tomography and MRI (15). In the present case, the findings from CEUS and enhanced MRI were highly consistent. If a patient with a low-level of AFP and no history of viral hepatitis presents with the aforementioned imaging characteristics, a diagnosis of PHSC should be considered. Specifically, central necrosis and peripheral enhancement are considered to be among the most valuable diagnostic characteristics of PHSC (15), and vascular invasion is another common diagnostic feature.

An exact diagnosis of PHSC depends on a number of pathological and immunohistochemical observations. Histologically, PHSC lesions contain spindle- and epithelial-cell components. The spindle-cell components consist of spindle-shaped cells, which exhibit oval and elongated nuclei with conspicuous nucleoli and spindle-shaped eosinophilic cytoplasms (13). Furthermore, these spindle cells form interlacing bundles and a local storiform pattern, and mitotic figures are frequently observed. Using immunohistochemical staining, the detection of $\mathrm{CK}$, epithelial membrane antigen (EMA) and vimentin may be useful for the diagnosis of PHSC. CK is regarded as an epithelial marker, while vimentin is a mesenchymal marker. Sarcomatoid lesions are positive for $\mathrm{CK}$ and vimentin. CK8 has been reported to be among the most critical indicators of SC (16). In addition, a number of other markers, including anion exchanger (AE) 1/AE3, 34ßE12, CAM 5.2, c-Kit, S-100 protein, HHF-35, kinesin-like protein-1, CD34 and HAM-56, may indicate a positive diagnosis of PHSC in certain cases. In the present case, the patient tested positive for $\mathrm{CK} 8$, vimentin and Ki-67 detection rate. Genetically, p53 has been shown to accumulate in the nuclei of undifferentiated spindle cells, indicating that the p53 gene mutation may be common in these cells (17). Thus, the mutation of the p53 gene may be involved in the progression of SC. Due to the complexity of the diagnosis, methods of differentiating PHSC from other types of liver sarcoma, such as chondrosarcoma, rhabdomyosarcoma, fibrosarcoma, leiomyosarcoma and malignant fibrous histiocytoma, are required. Tests for $\mathrm{CK}$ and EMA in these tumors are usually negative. In the present case, a distinction was made primarily between metastatic gastrointestinal stromal tumors (GISTs) and synovial sarcoma. These tumors are spindle-cell tumor lines with comparable morphology; however, the majority of GISTs express c-Kit protein and exhibit activating mutations in the KIT or PDGFRA proto-oncogenes (18). Furthermore, $>95 \%$ of synovial sarcomas (SSs) are characterized by the reciprocal chromosomal translocation $\mathrm{t}(\mathrm{X} ; 18)(\mathrm{p} 11.2 ; \mathrm{q} 11.2)$, which results in an SS-specific SYT-SSX fusion gene (19). In the present case, tests for the KIT gene and the SS18 gene mutation were negative, and the final diagnosis was PHSC.

The preferred treatment for PHSC is surgical resection. As this tumor type is rare and there are few large-sample studies, the exact effect of radiotherapy and chemotherapy is not clear. The prognosis of PHSC is particularly poor due to its high malignancy and local recurrence rates, venous invasion and intrahepatic, distant and lymph node metastasis (8). In particular, lymph node metastasis occurs almost twice as frequently in cases of PHSC as it does in HCC without sarcomatous change (14). The patient described in this study presented with recurrence of intrahepatic metastasis and venous invasion within 1 year. Extrahepatic metastasis can occur in the skin, pelvis, lungs and bladder, amongst other organs (20). The survival curve of patients with PHSC following hepatic resection is significantly worse than that of patients with ordinary HCC (8). The median survival time and 3-year survival rate of patients with SC have been found to be 9.6 months and $17 \%$, respectively (21). A Ki-67 proliferative index $>35 \%$ in patients with $\mathrm{SC}$ has been associated with poor prognosis (4); in the present case, the Ki-67 index was found to be $50 \%$.

In conclusion, PHSC is an aggressive tumor type, which is characterized by a rapid clinical course; a prompt diagnosis using histological and immunohistochemical analysis is therefore required. Early detection and treatment through appropriate examination and radical resection may improve patient prognosis. The present case of a rare, pure PHSC may improve the current understanding of this tumor type.

\section{Acknowledgements}

This study was supported by a grant from the National Natural Science Foundation of China (no. 81271585). 


\section{References}

1. Morgan MB, Purohit $\mathrm{C}$ and Anglin TR: Immunohistochemical distinction of cutaneous spindle cell carcinoma. Am J Dermatopathol 30: 228-232, 2008.

2. Nonnis R, Paliogiannis P, Giangrande D, Marras V and Trignano M: Low-grade fibromatosis-like spindle cell metaplastic carcinoma of the breast: A case report and literature review. Clin Breast Cancer 12: 147-150, 2012.

3. Chrysikos D, Zagouri F, Sergentanis TN, et al: Mucinous tubular and spindle cell carcinoma of the kidney: A case report. Case Rep Oncol 5: 347-353, 2012.

4. Travis WD: Sarcomatoid neoplasms of the lung and pleura. Arch Pathol Lab Med 134: 1645-1658, 2010.

5. Lee SE and Park SY: Sarcomatoid carcinoma of the small intestine: A rare and highly aggressive tumor. J Korean Surg Soc 83: 321-324, 2012.

6. Viswanathan S, Rahman K, Pallavi S, et al: Sarcomatoid (spindle cell) carcinoma of the head and neck mucosal region: A clinicopathologic review of 103 cases from a tertiary referral cancer centre. Head Neck Pathol 4: 265-275, 2010.

7. Kakizoe S, Kojiro M and Nakashima T: Hepatocellular carcinoma with sarcomatous change. Clinicopathologic and immunohistochemical studies of 14 autopsy cases. Cancer 59: 310-316, 1987.

8. Maeda T, Adachi E, Kajiyama K, Takenaka K, Sugimachi K and Tsuneyoshi M: Spindle cell hepatocellular carcinoma. A clinicopathologic and immunohistochemical analysis of 15 cases. Cancer 77: 51-57, 1996.

9. Shinoda M, Mukai M, Tanabe M, Hashiguchi N, Oda M and Kitajima M: Spindle cell carcinoma of the intrahepatic bile duct in a patient with primary sclerosing cholangitis. J Gastroenterol 38 1091-1096, 2003

10. Nakanishi C, Sato K and Ito Y: Combined hepatocellular carcinoma and neuroendocrine carcinoma with sarcomatous change of the liver after transarterial chemoembolization. Hepatol Res 42: 1141-1145, 2012.

11. Koda M, Maeda Y, Matsunaga Y, Mimura K, Murawaki Y and Horie Y: Hepatocellular carcinoma with sarcomatous change arising after radiofrequency ablation for well-differentiated hepatocellular carcinoma. Hepatol Res 27: 163-167, 2003.
12. Da Ines D, Bailly A, Lannareix V, et al: Hepatocellular carcinoma with sarcomatous change: Prompt and fatal intraabdominal recurrence after liver transplantation. Gastroenterol Clin Biol 33: 590-593, 2009

13. Giunchi F, Vasuri F, Baldin P, Rosini F, Corti B and D'Errico-Grigioni A: Primary liver sarcomatous carcinoma: Report of two cases and review of the literature. Pathol Res Pract 209: 249-254, 2013.

14. Eriguchi N, Aoyagi S, Hara M, Okuda K, Fukuda S, Tamae T and Kanazawa N: Malignant sarcomatoid tumor of the liver: Report of a case. Surg Today 31: 170-173, 2001.

15. Koo HR, Park MS, Kim MJ, Lim JS, Yu JS, Jin H and Kim KW: Radiological and clinical features of sarcomatoid hepatocellular carcinoma in 11 cases. J Comput Assist Tomogr 32: 745-749, 2008.

16. Haratake $\mathbf{J}$ and Horie A: An immunohistochemical study of sarcomatoid liver carcinomas. Cancer 68: 93-97, 1991.

17. Murata M, Miyoshi Y, Iwao K, et al: Combined hepatocellular/cholangiocellular carcinoma with sarcomatoid features: Genetic analysis for histogenesis. Hepatol Res 21: 220-227, 2001

18. Agaram NP, Baren A, Arkun K, Dematteo RP, Besmer P and Antonescu CR: Comparative ultrastructural analysis and KIT/PDGFRA genotype in 125 gastrointestinal stromal tumors. Ultrastruct Pathol 30: 443-452, 2006.

19. Kawauchi S, Ihara K, Nishikawa K, Sugino N, Takahashi M and Sasaki K: Synovial sarcoma arising in the vulva cytogenetically confirmed by SYT break-apart rearrangement fluorescence in situ hybridization: A case report and discussion of diagnostic methods. Oncol Lett 4: 955-959, 2012.

20. Nishie W, Iitoyo M, Koshiyama T and Kusama T: Sarcomatoid carcinoma of the liver with skin and pleural metastases. $\mathrm{Br}$ J Dermatol 148: 1069-1071, 2003.

21. Wang QB, Cui BK, Weng JM, Wu QL, Qiu JL and Lin XJ: Clinicopathological characteristics and outcome of primary sarcomatoid carcinoma and carcinosarcoma of the liver. J Gastrointest Surg 16: 1715-1726, 2012. 